\title{
VIDEO RETARGETING: A VISUAL-FRIENDLY DYNAMIC PROGRAMMING APPROACH
}

\author{
Zheng Yuan*, Taoran Lu* ${ }^{*}$ Yu Huang ${ }^{\dagger}$, Dapeng Wu*, Heather Yu ${ }^{\dagger}$ \\ * Dept. of ECE, University of Florida ${ }^{\dagger}$ Huawei Technologies Co. Ltd. USA
}

\begin{abstract}
Video retargeting is the task of fitting standard-sized video into arbitrary screen. A compelling retargeting attempts to preserve most visual information of original video as well as deliver a temporally consistent retargeted view. To handle long video sequences, we perform the task on a shot/subshot basis. For each frame, a crop pane is determined to optimally select a region of interest as the retargeted frame in two stages: i.e. minimizing visual information loss (intra-frame consideration) to yield source and destination crop pane parameters at boundary frames and minimizing visual information loss accumulation under the visual inertness (inter-frame consideration) constraints to search for a smooth transition of crop pane across interior frames. The second minimization process is remodeled as the shortest-path problem in graph theory and the parametric transition of crop panes is solved by dynamic programming. Experiments demonstrate our approach preserves salient regions of original video whilst offering eye-friendly visual consistency.
\end{abstract}

Index Terms - Video retargeting, spatial-temporal saliency, dynamic programming, brutal force search, shot detection

\section{INTRODUCTION}

As video processing chips become significantly powerful and compact, there emerges a large number of portable video broadcasting devices with small and customized screen size (e.g. iPhone, iPod, PSP) in consumer electronic market. This fact demands transplanting videos currently designed for computer, TV or DVD screens onto portable device platform to provide user easy access capability. The task of re-rendering video of industrial standardized size onto arbitrary screen size or aspect ratio is termed as video retargeting.

Available Approaches Resizing or Cropping are straightforward ways to perform video retargeting task. These approaches demand least computation but produce compromised results due to neglect of the difference of visual importance among different pixels. To preserve pixels with efficiency, seam carving [1], warping based [2], patch based [3] methods are proposed. Based on the generated saliency map, they rearrange pixels in target frames: the original geometric layout are faithfully maintained for adjacent pixels with higher visual saliency, while other less salient pixels are morphically squeezed to make up for the original-target screen size difference. These methods work well on still images because viewers tend to concentrate on those salient areas and generally tolerate the distortion of other areas with little interest; however, they become disastrous for videos because nearby retargeted frames are not necessarily morphically consistent. To avoid such problem, isotropic methods as single frame auto-cropping [4] [5] are proposed. They apply a crop pane to pan throughout each original frame to yield a

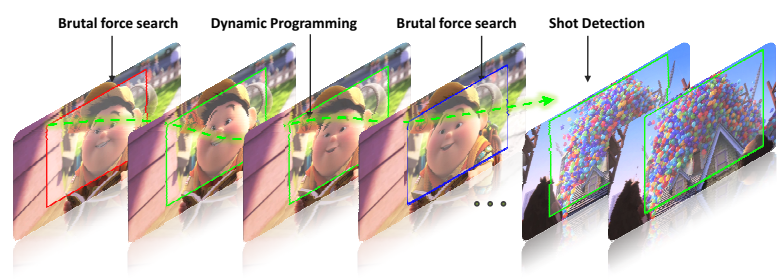

Fig. 1. video retargeting on a shot/subshot basis, green arrow: search for optimized trace of crop pane throughout a subshot using dynamic programming and brutal force search, red box: crop pane at start frame of a subshot, blue box: crop pane at end frame of a subshot.

region of interest with inside degradation minimized. Visual consistency is claimed by smoothing optimal crop pane parameters of each frame. This endeavor, nevertheless, does not do enough help to remove visual inconsistency along temporal axis because the crop pane parameters of adjacent frames are optimized independently and many twists and turns can still exist on the pane trace after smoothing. This suggests obvious frame jump back and forth, zoom in and out in the targeted video, which leads to viewer vertigo very soon. To carefully consider the visual experience along temporal axis, a back-tracing method [6] is presented to dynamically determine crop pane trace. This method adds another constraint to bound possible shift of crop panes among adjacent frames. It produces a retargeted video with frame consistency and thus avoids viewer discomfort. However, this method unfairly favors the initial parameter of crop pane of initial frame and clamp the crop panes of sequent frames near the initial value, i.e., the pane cannot crop/preserve salient objects soon as frame goes further, when the location of salient objects quite differs from that of the first frame. Thus this method cannot handle videos with frequent content motion.

Overview We propose an adaptive and content-aware cropping approach to retarget real life videos. Note that viewers are in fact not sensitive to abrupt crop pane change over adjacent frames with rapid scene change (shot boundaries). We first detect shots [7] [8] and then perform the task independently. A shot is then decomposed into multiple equal-length subshots for visual comfort and computational efficiency. For each frame, a 3-parameter (scale and location) rigid crop pane is determined to select a region of interest as retargeted frame. Within a shot, we optimally fix the scale of crop panes using proposed velocity-estimate method as otherwise a mild scale variation may cause significant visual degradation. Regarding the optimal location of crop pane, two boundary frames of each subshot are firstly processed. Aiming at keeping as much fidelity to original frame as possible, we search for locations of crop panes that minimize information loss function of the two frames, respectively and denote them the source and destination location of crop panes. Then 

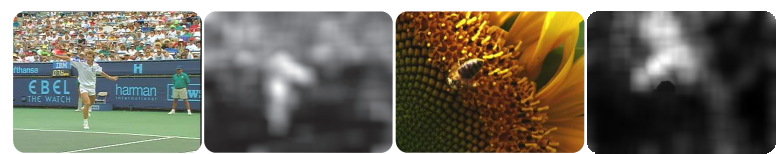

Fig. 2. Original frames and their saliency maps to denote the possibility of a pixel is attended by viewers

in order to seek optimal crop pane transition across interior frames, we address viewer visual expectations as intra-frame 'information fidelity' and inter-frame 'visual-inertness', which are two contradictory concerns. Accordingly, we minimize an accumulative penalty function as a total of both information loss and visual-inertness loss. Then finding the dynamic transition trace of rigid crop panes from source to destination locations is remodeled as a shortest path problem and is solved by dynamic programming. As subshot alternates each time, destination location is updated to synchronize the salient content of the last frame of next subshot (see Fig. 1). Thus as frame goes further, crop pane is not clamped onto the adjacency of source location as in [6] and is still capable of cropping salient objects of interest. Our retargeting results are free of shape distortion, have no annoying zoom in/out artifacts within the same scene, preserve the salient objects of interest throughout and visual consistency as well. The computational load of our method includes, Fourier Transform based saliency map calculation, brutal force search at boundary frames and dynamic programming for other frames, which is significantly lower than brutal force search for every frame as in [5][4].

\section{INTRA-FRAME VISUAL CONSIDERATIONS}

\subsection{Saliency Calculation}

Psychological studies [9] on human viewing experience concludes that viewers exhibit a remarkably discrimination on different regions in a screen. Generally, a region with salient pixels w.r.t their neighborhood is highly attended by viewer, which means various pixel offers different amount of visual information. Here we use saliency to denote the visual information of a pixel and wish to maximize the visual information accumulation preserved in the retargeted frame. In our paper [10] in ICIP'2010, we propose a non-linear method to generate spatio-temporal saliency. Eventually the saliency of each pixel is organized in a structure as "saliency map"(see Fig. 2), with the same size as original image.

\subsection{Intra-frame visual comfort measure: Information loss}

Within a frame, where visual consistency is isolated for the time being, the most reasonable expectation from viewer is that retargeted frame should present most fidelity to the original frame, i.e., the desired crop pane (with aspect ratio fixed) incurs least visual information loss of the original frame. A crop pane is indexed by three parameters, including scale $s$, upper-left corner's horizontal position $x$ and vertical position $y$. We crop an original frame from $(x, y)$ and resize the cropped content by $s$ times as the retargeted frame. This procedure does not cause geometric distortion because the aspect ratio of crop window remains constant all the time. However, cropping discards all visual information of pixels outside the crop pane while resizing degrades resolution by $s$ times inside crop pane. Thus we

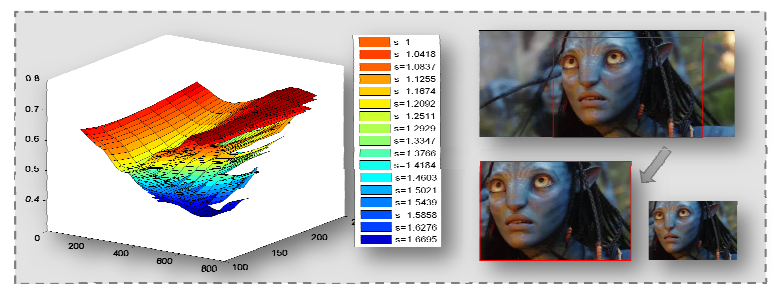

Fig. 3. Left: the shape of visual information loss function w.r.t $x, y$, s Right: retargeted frame is cropped and resized by a pane

define a Content-Aware information loss function as in Eq. 1

$$
\begin{gathered}
L=L_{c}\left(x, y, s W_{t}, s H_{t}\right)+\lambda \cdot L_{r}\left(x, y, s W_{t}, s H_{t}\right) \\
L_{c}\left(x, y, s W_{t}, s H_{t}\right)=1-\sum_{c=x, r=y}^{x+s W_{t}, y+s H_{t}} S M(r, c) \\
L_{r}\left(x, y, s W_{t}, s H_{t}\right)=\sum_{c=x, r=y}^{x+s W_{t}, y+s H_{t}}(I-\hat{I}(s))^{2}(r, c)
\end{gathered}
$$

where $L$ is total information loss, $L_{c}, L_{r}$ denote loss due to cropping and resizing. $S M$ is saliency map, $I$ is the original image, $x, y, s$ are the three parameters of a crop pane, $\hat{I}(s)$ is the scale degraded image created by downsampling $I$ to $s$ times smaller followed by upsampling to its original size (use repetition to upsample and a Gaussian filter is applied before upsampling to avoid alias). $\lambda$ is the trade-off of resizing vs. cropping, $W_{t}$ and $H_{t}$ are target width and height.

Our goal here is to find a desired crop pane $P(\hat{x}, \hat{y}, \hat{s})$ that corresponds to least information loss.

$$
P(\hat{x}, \hat{y}, \hat{s})=\arg \min _{x, y, s} L\left(x, y, s W_{t}, s H_{t}\right)
$$

Considering that humans are very sensitive to scale variation even at a modest level, we alternatively determine a good scale $s$ using the method in Sec. 4 and fix $s$ throughout a shot and search for optimal $x, y$ instead.

$$
P(\hat{x}, \hat{y})=\arg \min _{x, y} L\left(x, y, \hat{s} W_{t}, \hat{s} H_{t}\right)
$$

Given the non-linear property of the information loss function, we use brutal force search [10] to find the optimal $x, y$. Fig. 3 illustrate the shape of information loss function.

\section{INTER-FRAME VISUAL CONSIDERATIONS}

\subsection{Inter-frame visual comfort: Visual inertness}

A unique characteristic of video retargeting task as oppose to resizing still image is temporal coherence. Minimizing intra-frame visual information loss only makes resultant video suffer from annoying jitters due to independent but inconsistent crop pane parameters. Here we take into account the fact that viewers need a steady and smooth video content transition known as "visual inertness". Note that across adjacent frames, a shift of crop panes imposes artificial camera motion to retargeted frames. On one hand, an absolute free inter-frame shift makes it possible to crop and then preserve the most 


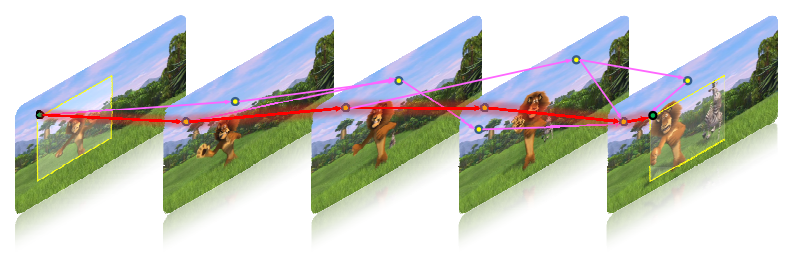

Fig. 4. Graph Model for optimize crop pane trace; green: source and destination nodes. yellow: candidate node for each frame. red: shortest path to denote optimized dynamic trace

salient region of each different frame. On the other hand, visual inertness favors a modest inter-frame shift or no shift at best. In our approach, we consider together these contradictory visual comfort clues and balance them. To measure the visual performance w.r.t the location of crop pane, we define a function of visual penalty accumulation within a subshot as in Eq. 4

$$
\begin{array}{r}
Q\left(\bar{x}_{N}, \bar{y}_{N}\right)=\sum_{i=1}^{N} L\left(x_{i}, y_{i}\right)+\omega \cdot \sum_{i=2}^{N} E I\left(x_{i-1}, y_{i-1}, x_{i}, y_{i}\right) \\
E I\left(x_{i-1}, y_{i-1}, x_{i}, y_{i}\right)=\left(x_{i}-x_{i-1}\right)^{2}+\left(y_{i}-y_{i-1}\right)^{2}
\end{array}
$$

where $L$ is intra-frame visual information loss of frame $i, E I$ is temporal penalty that constrains shift of panes across adjacent frames. $x_{i}, y_{i}$ is the location of upper-left corner of crop pane of frame $i$ and $N$ is the total number of frames in a subshot. $\left(\bar{x}_{N}, \bar{y}_{N}\right)$ is a dynamic trace of the upper-left corner of crop pane over a subshot and $\omega$ is the trade-off of two visual concerns. Our goal is to find the optimal trace $\left(\hat{\overline{x_{N}}}, \hat{\overline{y_{N}}}\right)$ such that $Q$ is minimized.

\subsection{Dynamic Programming Solution}

We model the solution space $\left(\bar{x}_{N}, \bar{y}_{N}\right)=\left\{x_{i}, y_{i}\right\}_{i=1}^{N}$ by a graph illustrated in Fig. 4, where each node $\left(x_{i}, y_{i}\right)$ denotes the upper-left corner location of a candidate crop pane of frame $i$ and each edge $\left(x_{i-1}, y_{i-1}\right) \rightarrow\left(x_{i}, y_{i}\right)$ represents the shift of crop pane from frame $i-1$ to frame $i$. The cost on each node is visual information loss $L\left(x_{i}, y_{i}\right)$ and as for each edge, the cost corresponds to temporal penalty $E I\left(x_{i-1}, y_{i-1}, x_{i}, y_{i}\right)$. Thus minimizing $\mathrm{Q}$ in Eq. 4 is equivalent to finding the shortest path from node $\left(x_{1}, y_{1}\right)$ to $\left(x_{N}, y_{N}\right)$. The optimization can be easily solved by Dynamic programming. The recursive format of the objective function in Eq. 4 is as follows,

$$
\begin{aligned}
Q\left(\bar{x}_{i}^{k}, \bar{y}_{i}^{k}\right) & =\min _{j}\left\{Q\left(\bar{x}_{i-1}^{j}, \bar{y}_{i-1}^{j}\right)+\right. \\
& \left.\omega \cdot E I\left(x_{i-1}^{j}, y_{i-1}^{j}, x_{i}^{k}, y_{i}^{k}\right)\right\}+L\left(x_{i}^{k}, y_{i}^{k}\right)
\end{aligned}
$$

where $Q\left(\bar{x}_{1}^{1}, \bar{y}_{1}^{1}\right)=0, Q\left(\bar{x}_{i}^{k}, \bar{y}_{i}^{k}\right)$ denotes minimized cost accumulation or equivalently the shortest path from source node $\left(x_{1}^{1}, y_{1}^{1}\right)$ of frame 1 to the $k$ th node of frame $i . Q\left(\bar{x}_{i-1}^{j}, \bar{y}_{i-1}^{j}\right)$ is the shortest path up to the $j$ th node of frame $i-1, E I\left(x_{i-1}^{j}, y_{i-1}^{j}, x_{i}^{k}, y_{i}^{k}\right)$ denotes the cost of edge connecting the $j$ th node with frame $i-1$ to the $k$ th node of frame $i$ and $L\left(x_{i}^{k}, y_{i}^{k}\right)$ is the cost of the $k$ th node of frame $i$.

Given the source and destination node, Algorithm 1 describes the procedure to find the shortest path between them. As mentioned before, a shot is divided into equal-length subshots. We assign destination as the optimized crop pane location in Eq. 3 and source as the

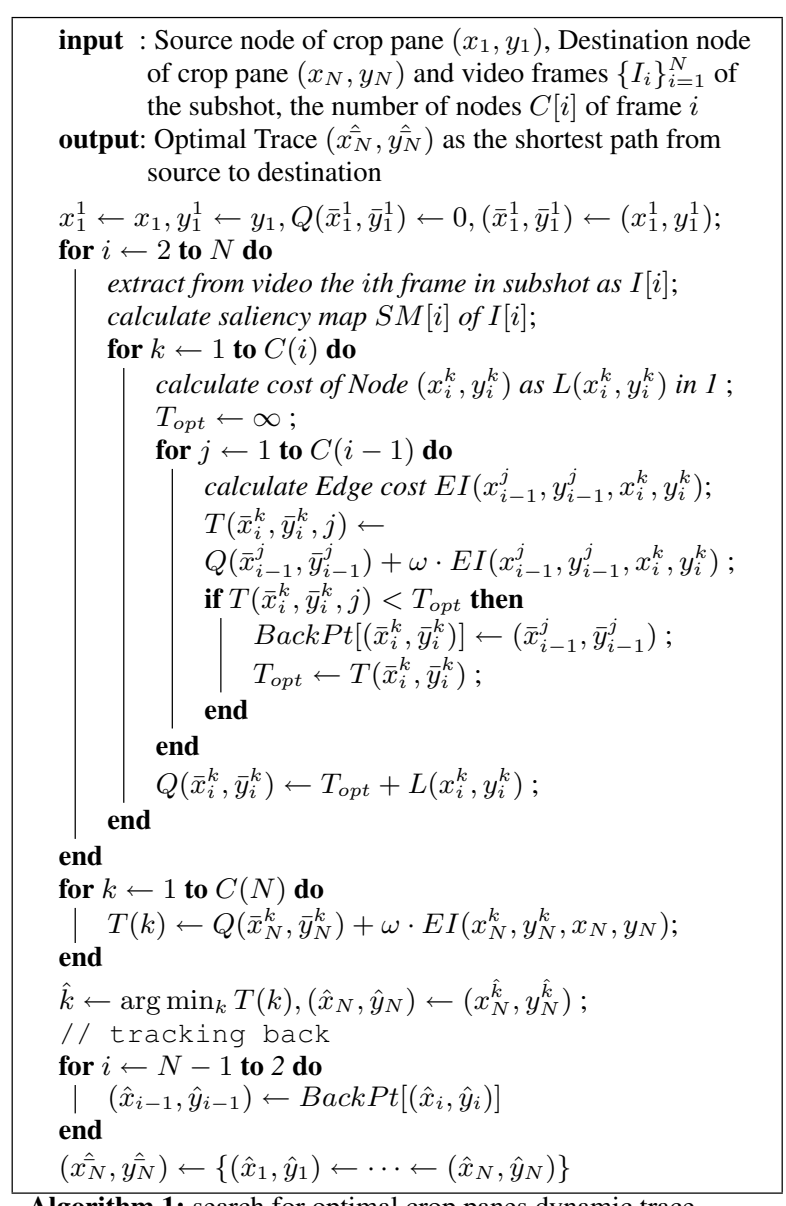

Algorithm 1: search for optimal crop panes dynamic trace

destination of previous subshot to avoid jitter between subshots. By this measure, every subshot, we update crop pane to a free position that crops most salient area at that time. Meanwhile, dynamic programming method yields a smooth crop pane transition trace over each subshot. So with consistent views, our method can always retarget salient region no matter how fast it moves in a shot.

\section{OPTIMIZE SCALE IN A SHOT}

The optimal scale which minimizes information loss function in Eq. 3 depends on weight $\lambda$. Generally, $\lambda$ is specified according to aesthetic preference of viewers and it is quite subjective among different viewers. Most scenes of movies, news or commercials portrait foreground salient objects in high resolution. So we assume that most viewers are more expecting a crop pane with complete objects in as people usually prefer a global view with broad visible range at the price of resolution rather than only access to a limited area. Here resizing is more preferred to cropping. On the contrary, in most farview scenes (e.g.soccer broadcasting) where salient objects occupies small areas, most viewers would like to focus on and track the object rather than huge resolution degradation, otherwise, objects become 
too small to recognize. Here cropping becomes more preferable than resizing. Based on aesthetic requirement, we specify an initial weight $\lambda$ and find optimized scales of some sampled frames based on Eq. 2 and average them as the scale of the shot. Mostly, this simple method works fine, however, when a salient object is moving fast, the crop pane may not move fast enough to catch up with the object due to visual consistency constraint. This leads to cut off some parts of the object and it suggests a larger scale of crop pane needed. We use the velocity of dynamic crop pane transition within a shot to estimate how fast objects of interest move. Then based on the velocity estimate, we adjust the weight $\lambda$ in order to obtain a larger scale, which yields a larger crop pane to include salient objects completely.

$$
\lambda^{\prime}=\left(1+\exp \left\{-\left|\frac{1}{N} \cdot \sum_{i=1}^{N} \frac{\left(\hat{x}_{i}-\hat{x}_{i-1}\right)^{2}+\left(\hat{y}_{i}-\hat{y}_{i-1}\right)^{2}}{L_{i}^{2}}-v_{\alpha}\right|\right\}\right)^{-1}
$$

where $\frac{1}{N} \cdot \sum_{i=1}^{N} \frac{\left(\hat{x}_{i}-\hat{x}_{i-1}\right)^{2}+\left(\hat{y}_{i}-\hat{y}_{i-1}\right)^{2}}{L_{i}^{2}}$ is the velocity estimate of crop pane transition, $N$ is the total number of frames in the shot. $L_{i}$ is the maximum distance a crop window can move from $\left(x_{i-1}, y_{i-1}\right)$ and $v_{\alpha}$ denotes a reference velocity. Given the updated weight $\lambda^{\prime}$, a new scale average is optimized for the shot. Then we start over to find optimal trace of crop pane under the new scale.

\section{EXPERIMENTS}

We implement our approach in C++ using OpenCV and FFTW3 library. The test is carried out on a diversity of video types, including movie, entertainments, news, sports, etc. Original videos can be in any screen size, length and also viewers are allowed to specify any retargeted smaller screen display size or customized aspect ratio. In order to present real visual experience, we put quite a number of video retargeting results at our website ${ }^{1}$.

We also compare our approach with two baseline isotropic retargeting methods: single frame smoothing(SFS) [4][5] and back tracking (BT) [6]. Since there are no available executables for them, we implement SFS and BT according to the descriptions of [5] and [6], respectively. We run the three approaches on two cartoons and one fashion show. For dynamic evaluation of the retargeting performances and corresponding analysis, please visit our website. Generally, SFS suffers from jitter and viewers will feel vertigo soon. BT is mostly acceptable, however, the retargeted video is not always able to preserve salient region of interest in original video. In comparison, our method throughout preserves salient region as frame goes further and avoids jitter effects as well.

Fig. 5 presents result comparison in a static fashion. We illustrate crop panes on original frames with frame number noted in Fig. 5. Original video is in resolution $640 \times 352$ and the specified retargeted size is $320 \times 240$. An initial weight $\lambda$ (cropping/resizing preference) is provided as 0.3 and the subshot length is 120 frames. In results of SFS, although lion and zebra are preserved completely, the crop pane shifts back and force frequently, which means huge jitter effects in retargeted video. In results of BT, at the beginning frame \#49 to \#143, the crop pane includes complete zebra; however, as frame goes to \#264 until \#379, the crop pane is left behind by zebra's fast swirling and thus cuts zebra outside in retargeted video. In contrast,

\footnotetext{
${ }^{1}$ http://www.mon.ece.ufl.edu/public/ZhengYuan/retargeting.htm
}
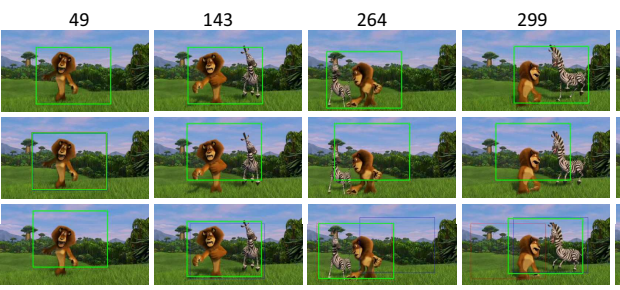

379

Fig. 5. Retargeting Results: top: single frame search and smoothing (SFS), middle: back tracking (BT), bottom: the proposed approach

our result yields a visual consistent crop pane trace to preserve zebra completely.

\section{CONCLUSIONS}

We propose a content-aware approach to fit video sequence into arbitrary screen size. For each video frame, a crop pane is applied to select a region of interest as the retargeted frame. The pane aims to preserve maximized visual information of original video under temporal visual consistency constraint. A dynamic programming solution is presented to optimize the temporal trace of crop pane. We also introduce subshots to make trade-off between intra-frame visual comfort and contradictory inter-frame comfort. Experiment results show that our approach can preserve salient region throughout the video no matter how frequent the salient content moves and offer visual consistency as well. Noticeably the weight of resizing vs. cropping affects video retargeting results significantly. In the near future, we will explore its quantitative relation with aesthetic preference and extend our approach to be applicable to any type of videos with a diversity of aesthetic preferences.

\section{REFERENCES}

[1] M. Rubinstein and et al., "Improved seam carving for video retargeting," ACM Trans. on Graphics, 2008.

[2] L. Wolf, M. Guttmann, and D. Cohen-Or, "Non-homogeneous content-driven video-retargeting," in ICCV, 2007.

[3] D. Simakov, Y. Caspi, and et al., "Summarizing visual data using bidirectional similarity," CVPR, 2008.

[4] F. Liu and M. Gleicher, "Video retargeting: automating pan and scan," in ACM International Conf. on MultiMedia, 2006.

[5] G. Hua and et al., "Efficient Scale-space Spatiotemporal Saliency Tracking for Distortion-Free Video Retargeting," in ACCV, 2009.

[6] T. Deselaers, P. Dreuw, and H. Ney, "Pan, Zoom, Scan-Timecoherent, Trained Automatic Video Cropping," in CVPR, 2008.

[7] J. Boreczky and et. al, "Comparison of video shot boundary detection techniques," J. of Electronic Imaging, 1996.

[8] N.V. Patel and I.K. Sethi, "Video shot detection and characterization for video databases," Pattern Recognition, 1997.

[9] L. Itti and et al., "A model of saliency-based visual attention for rapid scene analysis," IEEE Trans. PAMI, 1998.

[10] T. Lu, Z. Yuan, and et al., "Video Retargeting with nonlinear spatio-temporal saliency fusion," in ICIP, 2010. 